\title{
Evaluación de indicadores de atención en el Servicio de Admisión Continua: tiempo de demora en ingreso y tiempo de permanencia
}

\author{
Héctor Zamarripa-Gutiérrez, Óscar del Ángel-Guevara(†), Erick Crespo-Solís, \\ Ramón Martínez-Hernández, Juan Jesús Maldonado-Muñoz y Sylvia González-Esparza*
}

Servicio de Admisión Médica Continua, Hospital Regional de Alta Especialidad de Ciudad Victoria Bicentenario 2010, Ciudad Victoria, Tamps., México

\begin{abstract}
RESUMEN
La calidad de la atención en los Servicios de Urgencias o Admisión Continua es una de las misiones del Hospital Regional de Alta Especialidad de Ciudad Victoria. Con el propósito de contar con un estándar de evaluación basado en indicadores de calidad nacional, adoptamos los dos principales indicadores que evalúan el tiempo de espera para la atención y el tiempo de estancia en sala de observación. El objetivo fue medir el tiempo para establecer un plan terapéutico adecuado para el paciente, por medio de auditoría clínica. En la auditoría se encontraron factores que impiden la adherencia a estos indicadores y se implementaron las mejoras necesarias para su cumplimiento. Se realizaron tres mediciones, con la evaluación de 42, 53 y 48 casos respectivamente. La estandarización y medición de los indicadores de atención en las áreas críticas, como Admisión Médica Continua, por medio de auditoría clínica permiten conocer los procesos e identificar problemas, y de esta manera aplicar las acciones de mejora con efecto permanente, así como una mejor coordinación en los niveles de atención.
\end{abstract}

Palabras clave: Indicadores. Calidad. Mejoras. Urgencias. Admisión Médica Continua. Satisfacción. Tiempo de espera.

\begin{abstract}
The quality of care in the emergency and continuous admission departments is one of the missions of the High Specialty Regional Hospital in Ciudad Victoria, Tamaulipas, Mexico. To have an evaluation standard based on national indicators of quality of care, we conducted a clinical audit by adopting two main indicators, the waiting time to receive care and the time spent in the observation room, in order to measure the time taken to establish an adequate therapeutic plan for the patient. Three measurements were made in which we evaluated 42,53 and 48 cases, respectively. In the audit, we found factors that prevented adherence to these indicators, and the necessary improvements
\end{abstract}

\section{Correspondencia:}

*Sylvia González-Esparza

Hospital Regional de Alta Especialidad de Ciudad Victoria Bicentenario 2010

Libramiento Guadalupe Victoria, s/n

Área de Pajaritos

Date of reception: $12-04-2018$

C.P. 87087, Ciudad Victoria, Tamps., México

Date of acceptance: 06-06-2018

E-mail: dra_sylvia_glz@ $@$ hotmail.com

DOI: 10.24875/HMCM.18000117 
were implemented to comply with them. The standardization and measurement of indicators of care through a clinical audit in critical areas such as continuous medical admission allowed us to understand the processes involved and identify existing problems. Thus, we could implement actions for improvement with a permanent effect, as well as achieve a better coordination between the levels of care. (Hosp Med Clin Manag. 2018;11:56-63)

Corresponding author: Sylvia González-Esparza, dra_sylvia_glz@ hotmail.com

Key words: Indicators. Quality. Improvements. Emergencies. continuous medical admission.satisfaction. Waiting time.

\section{INTRODUCCIÓN}

Dentro de las funciones principales del Servicio de Admisión Continua Hospitalaria orientadas a la atención oportuna de los pacientes están el promover la recepción inmediata del paciente, estabilizarlo y realizar los estudios de gabinete o procedimientos necesarios para su recuperación; por lo que se realizaron varios protocolos de auditoría clínica para evaluar si el proceso clínico de atención se ajusta a las medidas establecidas en la calidad de la atención, tomándose para ello como herramienta el ejercicio de entrega de guardia, sin éxito, ya que fueron detectados hallazgos como estancia prolongada de pacientes en sala de Admisión Médica Continua, así como parte de la entrega-recepción de pendientes a pacientes por valorar en sala de espera. Esto generó inconformidad entre el personal médico, la cual fue manifestada en Sesiones de Comité de auditoría clínica; estableciéndose entonces como objetivo cambios en la perspectiva en la auditoría en la búsqueda de parámetros que evalúen la calidad de atención en el Servicio de Admisión Continua. En el Catálogo de Indicadores de Atención Médica De Alta Especialidad se especifican los siguientes indicadores: razón de urgencias calificadas atendidas, porcentaje de internamiento a urgencias, porcentaje de ingresos a hospitalización por urgencias y porcentaje de ocupación en urgencias ${ }^{1}$; los cuales corresponden a indicadores de actividad y resultado, pero no evalúan la calidad mediante parámetros como los tiempos aceptados para su proceso.

Dependiendo del concepto que interese medir, los indicadores pueden ser de dos tipos: de demanda, también conocidos como de cantidad, y de calidad de las actividades. Dentro de los de calidad, nos hemos centrado en dos aspectos: indicadores de proceso (aquellos que evalúan la calidad de los procesos asistenciales, es decir, cómo se hacen las cosas) e indicadores de resultado (los que miden el resultado final de las actividades sanitarias, independientemente del modo como estas hayan sido hechas).

Se establecen por tanto tres grupos de indicadores:

- Indicadores de actividad. Son aquellos que miden la cantidad de solicitudes de asistencia que se presentan en el Servicio de Urgencias. Tienen un valor informativo para evaluar el uso de los recursos asistenciales y su gestión, además de permitir una comparación de la actividad del centro con los Servicios de Urgencias de otros centros.

- Indicadores de calidad de proceso. Son aquellos que miden aspectos cualitativos del proceso de asistencia de la urgencia. Estos indicadores miden el funcionamiento del Servicio de Urgencias desde el punto de vista organizativo y de la calidad de los procesos.

- Indicadores de resultado. Son aquellos que miden los resultados finales de las actividades asistenciales. Informan, por tanto, de la calidad y capacidad técnica y resolutiva del Servicio de Urgencias ${ }^{2,7}$.

Por lo que, en consenso para la implementación de esta auditoría, se tomaron los indicadores de calidad de proceso y de resultados como herramienta para evaluar nuestra asistencia en el Servicio de Admisión Médica Continua (SAMC) enfocándonos en dos indicadores: 1) tiempo medio para la atención en Admisión Continua y 2) tiempo medio de permanencia en Admisión Continua $^{7}$, con el propósito de implementar mejoras que disminuyan la espera del usuario para la atención y la estancia prolongada en el SAMC.

\section{Consideraciones éticas}

El estudio no infringió ningún derecho del paciente y la privacidad o confidencialidad de sus datos estuvo 
garantizada. No se recogió ningún dato que pudiera ser utilizado para identificar al paciente o a algún practicante. La auditoría no supuso ningún riesgo ni cargo para el paciente mas allá de su rutina de atención. Los datos fueron registrados por personal que acceden a ellos de manera habitual. No se obtuvo información del paciente diferente a la necesaria para su atención. La participación del paciente no representó en ningún caso un conflicto potencial de obligación con este, como alguna compensación entre el costo y calidad o su seguridad. La auditoría no constituyó un riesgo o una carga más para el paciente, en el sentido de consumir tiempo o requerir alguna información sensible. No se realizaron intervenciones clínicas ni se emplearon sistemas no aprobados, así como tampoco se demandó la realización de intervenciones diferentes entre el grupo de pacientes o el personal.

\section{Objetivos}

Los objetivos de la auditoría fueron los siguientes:

1. Evaluar y estandarizar los procedimientos necesarios para mantener el periodo entre la recepción y atención del paciente, y el periodo de estancia en el SAMC, dentro de lo establecido por norma y la Guía de práctica clínica para triaje.

2. Evaluar y estandarizar la coordinación de los servicios de especialidad en la atención de los pacientes del SAMC, evitando esperas innecesarias por cuestiones organizativas, coordinando las acciones provenientes de los distintos Servicios del hospital.

3. Establecer la metodología (o protocolo) para el correcto registro de información de los indicadores de funcionamiento y producción en el área de Admisión Médica Continua.

\section{MATERIAL Y MÉTODOS}

\section{Descripción de la muestra}

Se incluye la revisión de registro en bitácora de solicitud de atención en ventanilla y la revisión del expediente clínico electrónico de pacientes que solicitan atención por el SAMC en el periodo comprendido entre el 12 de abril y el 31 de mayo del 2017 para la primera medición, del 2 al 25 de agosto de 2017 para la segunda medición, y del 22 de octubre al 7 de noviembre del mismo año para la tercera medición.

Se excluyeron los casos de pacientes recibidos por referencia de otras unidades para la medición basal y, además, por consenso se determinaron como criterios de exclusión la ausencia de nota médica o registro de expediente clínico electrónico atribuible a egreso voluntario para la segunda y la tercera medición.

\section{Muestra y periodo de la auditoría clínica}

Para la primera medición se revisaron 41 casos seleccionados aleatoriamente de los turnos matutino y vespertino, en el periodo comprendido entre el 12 de abril y el 31 de mayo del 2017 para el primer indicador y 36 para el segundo indicador.

Para la segunda medición se revisaron 48 casos para el primer indicador y 40 para el segundo indicador, en el periodo comprendido entre el 2 y el 25 de agosto de 2017.

Para la tercera medición se revisaron 48 casos seleccionados aleatoriamente de los todos los turnos, que aplicaron para los dos indicadores, en el periodo del 22 de octubre al 7 de noviembre del 2017.

\section{Estrategia de recolección de datos}

Concurrente.

\section{Fuente de datos}

Se realizó una cédula de recolección de datos obtenidos del registro de los usuarios en la bitácora de ventanilla y la sala de observación, así como la revisión de expediente clínico electrónico.

\section{Medidas para la auditoría}

Se presentan dos indicadores que pretenden establecer un sistema común, homogéneo y fiable de información en el SAMC, que reflejan de un modo claro, fiable y significativo los perfiles básicos de la actividad asistencial, sin perjuicio de dejar abierta la posibilidad de estudiar y aceptar sugerencias que tiendan a completarlo y perfeccionarlo. 


\section{Tiempo medio para la atención en Admisión Continua}

Fundamento: uno de los aspectos clave en la calidad de la atención urgente, tanto desde el punto de vista clínico como del de la calidad percibida por los usuarios, es la rapidez con la que un médico atiende y evalúa al paciente cuando este acude por un problema que considera urgente. Este indicador permite conocer la rapidez (o lentitud) con la que se comienza a prestar atención por un médico y, por tanto, la eficacia y eficiencia de la organización del proceso para evitar que las patologías graves se vean afectadas negativamente por demoras en la asistencia inicial.

Expresión: se expresa como minutos de media, en relación al triaje hospitalario del Servicio de Admisión Continua basado en la codificación de tres colores ${ }^{6}$ (Fig. 1).

Criterios de exclusión: se excluyeron del cómputo de este indicador los casos en los cuales la consulta fue anulada o interrumpida, y aquellos otros en los que el usuario desistió de consultar y se ausentó; también los casos en los que no existía constancia o registro de los datos horarios, fuesen de la llegada o de la consulta, así como los pacientes recibidos por referencia previamente notificada.

\section{Tiempo medio de permanencia en Admisión Continua}

Descripción: tiempo total que permanece el paciente en el SAMC desde que llega hasta que abandona el mismo, sea por alta, por ingreso, por fallecimiento, por traslado a una unidad de observación o por traslado a otro centro.

Fundamento: una característica fundamental de la atención urgente es la rapidez. El paciente que es visto en el SAMC debe ser atendido sin grandes dilaciones, con el objetivo de identificar en poco tiempo su problema y buscarle la solución más adecuada en el contexto real en que se presenta. Un tiempo de estancia prolongado puede significar que existen déficits de coordinación, circuitos inadecuados, servicios de soporte insuficientes, etc. En este caso, cada hospital debería establecer sus indicadores internos para averiguar las causas del incremento de la permanencia ${ }^{2,5}$. Por lo que, en consenso durante las reuniones de Comité y mesas de trabajo

\begin{tabular}{|l|}
\hline $\begin{array}{l}\text { Si usted es clasificado color rojo, su atención } \\
\text { será inmediata y continua. }\end{array}$ \\
$\begin{array}{l}\text { Si usted es clasificado color amarillo, su tiempo } \\
\text { de espera será de } 15 \text { a } 30 \text { min como máximo. }\end{array}$ \\
$\begin{array}{l}\text { Si usted es clasificado color verde, se le } \\
\text { atenderá en un máximo de } 1 \text { a } 4 \mathrm{~h} .\end{array}$ \\
\hline
\end{tabular}

Figura 1. Codificación del triaje hospitalario. Triaje hospitalario de primer contacto en los Servicios de Urgencias de adultos para el segundo y tercer nivel. GPC. $\mathrm{N} . \stackrel{\circ}{\text { de }}$ registro: ISSSTE-339-08.

de auditoría clínica, por observancia de cuerpo directivo se acordó que, para esta unidad, este periodo no debía extenderse más allá de ocho horas.

La NOM-027-SSA3-2013, Regulación de los servicios de salud, que establece los criterios de funcionamiento y atención en los Servicios de Urgencias de los establecimientos para la atención médica establece que «5.6 Los pacientes no deberán permanecer más de 12 horas en el servicio de urgencias por causas atribuibles a la atención médica. Durante ese lapso, se deberá establecer un diagnóstico presuntivo, su manejo y pronóstico inicial, con la finalidad de que el médico determine las posibles acciones terapéuticas que se deberán llevar a cabo dentro y fuera de dicho servicio, para la estabilización y manejo del paciente» ${ }^{4}$.

La permanencia de los pacientes en los Servicios de Urgencias no deberá exceder por razones médicas las cuatro horas en hospitales de segundo nivel con una a tres especialidades básicas y las ocho horas en hospitales generales de segundo nivel con cuatro especialidades y hospitales de tercer nivel; en este tiempo se dará el abordaje inicial y se determinará si el paciente debe ser ingresado a hospitalización, transferido a otro nivel resolutivo, referido a la consulta externa o a su unidad de origen, o egresar como alta a su domicilio ${ }^{3,4}$.

Expresión: minutos de permanencia media.

Criterios de exclusión: se excluyeron del cómputo de este indicador los casos en los cuales la consulta fue anulada o interrumpida, y aquellos otros en los que el usuario desistió de consultar y se ausentó. Igualmente se excluyeron los casos en los que no existía constancia o registro de los datos horarios, fuesen de la llegada o de la consulta. 
Tabla 1. Primera medición. Periodo: 12 de abril al 31 de mayo del 2017

\begin{tabular}{llccc}
\hline Parámetro & Evidencia de calidad (y excepciones o gestión crítica) & Evidencia de calidad & $\%$ esperado & $\%$ efectivo \\
\hline 1 & Tiempo medio para la atención en Admisión Continua & $30 / 41$ & 100 & 73.1 \\
2 & Tiempo medio de permanencia en Admisión Continua & $36 / 36$ & 100 & 100 \\
\hline
\end{tabular}

Tabla 2. Codificación de triaje para la primera medición

\begin{tabular}{lcc}
\hline Código & Casos en rango & Casos fuera de rango \\
\hline Inmediato & $1(2.4 \%)$ & \\
15 a 30 minutos & $10(24.3 \%)$ & $6(14.6 \%)$ \\
1 a 4 horas & $19(43.3 \%)$ & $5(12.1 \%)$ \\
\hline
\end{tabular}

\section{ANÁLISIS DE LA PRIMERA MEDICIÓN}

En la primera medición se evaluaron 42 casos, que fueron seleccionados aleatoriamente de los turnos matutino y vespertino, del periodo comprendido entre el 12 de abril y el 31 de mayo del 2017, sin excepciones.

Por consenso del Comité de auditoría clínica se determinaron como criterios de exclusión: la ausencia de nota médica o registro de expediente clínico electrónico atribuible a egreso voluntario o que no se requiriese nota médica por procedimiento (aplicación de medicamentos, curaciones, férulas), siendo excluido solo un caso por ausencia de nota médica y quedando así 41 casos con criterios para evaluación para el indicador 1 y 36 casos para el indicador 2 .

Para el indicador 1, se obtuvo evidencia de calidad en el tiempo medio para la atención en Admisión Continua en 30/41 casos, es decir, un $73.1 \%$ de efectividad frente al $100 \%$ esperado (Tabla 1). De estos casos, 6/41 (14.6\%) se encontraron fuera del rango de tiempo de espera para atención establecido para triaje, que en su caso era el código amarillo (Tabla 2).

Para el indicador 2, se obtuvo una evidencia de calidad en el tiempo medio de permanencia en Admisión Continua en 36/36 casos, una efectividad del 100\% (Tabla 1), lo que es de gran importancia sostener para mantener la alta calidad del Servicio para este indicador.

Los problemas detectados fueron: la falta de capacitación en triaje del personal de primer contacto; la falta de un buen sistema de registro de usuarios desde el primer contacto y su seguimiento; y que el personal de ventanilla es insuficiente para la cobertura de todos los turnos, ya que se cuenta únicamente con una trabajadora social, que en los turnos nocturnos y durante el fin de semana se apoya en personal administrativo para recibir al usuario, ya que es la única en su función para toda la unidad hospitalaria.

De los cinco pacientes de los cuales se desconoce destino, uno fue por alta voluntaria y se asume que los otros cuatro solo requirieron aplicación de medicamento o curación.

Propuestas de mejora:

- Se realizó capacitación del personal de primer contacto.

- Se estableció un registro en bitácora de solicitud de atención.

- Se acordó implementar comunicación efectiva entre el personal de trabajo social y el médico.

\section{ANÁLISIS DE LA SEGUNDA MEDICIÓN}

Se evaluaron 53 casos, seleccionados aleatoriamente, encontrándose solo registro en turnos matutino y vespertino, en el periodo comprendido entre el 2 de julio y el 25 de agosto del 2017.

Se excluyó a aquellos que se fueron sin avisar (3/53) y las altas voluntarias (2/53), así como a aquellos con datos incompletos por falta de registro (13/53) para evaluar tiempo de estancia, quedando un total de 48 para el indicador 1 y 40 para el indicador 2 .

Para el indicador 1, se obtuvo evidencia de calidad en el tiempo medio para la atención en Admisión Continua 
Tabla 3. Segunda medición. Periodo: 2 de julio al 25 de agosto del 2017

\begin{tabular}{llccc}
\hline Parámetro & $\begin{array}{l}\text { Evidencia de calidad } \\
\text { (y excepciones o gestión critica) }\end{array}$ & \% esperado & $\begin{array}{c}\text { \% efectivo } \\
\text { medición 1 }\end{array}$ & $\begin{array}{c}\text { \% efectivo } \\
\text { medición 2 }\end{array}$ \\
\hline 1 & Tiempo medio para la atención en Admisión Continua & $100 \%$ & 73.1 & 95.8 \\
2 & Tiempo medio de permanencia en Admisión Continua & $100 \%$ & 100 & 92.5 \\
\hline
\end{tabular}

Tabla 4. Codificación de triaje para la segunda medición

\begin{tabular}{lcc}
\hline Código & Casos en rango & Casos fuera de rango \\
\hline Inmediato & $3(6.25 \%)$ & \\
15 a 30 minutos & $8(16.6 \%)$ & $1(2 \%)$ \\
1 a 4 horas & $35(72.9 \%)$ & $1(2 \%)$ \\
\hline
\end{tabular}

en $46 / 48$ casos, es decir, un $95.8 \%$ de efectividad frente al $100 \%$ esperado (Tabla 3 ). De los cuales 2/48 (4.1\%) se encontraron fuera del rango de tiempo de espera para atención establecido por el triaje, un código amarillo y uno verde (Tabla 4), lo que es mas aceptable.

Para el Indicador 2, se obtuvo evidencia de calidad en el tiempo medio de permanencia en Admisión Continua en $37 / 40$ casos, reduciendo el resultado a una efectividad del 92.5\% (Tabla 3), lo que representa una disminución en la calidad del Servicio para este indicador.

Encontramos como causas de fallo para esta segunda medición, para el indicador 1 (tiempo medio para la atención en Admisión Continua):

- Médico de nuevo ingreso, que no contaba con capacitación adecuada para triaje y no estaba concienciado de la importancia del registro de información.

- Cada turno en ventanilla (trabajo social y personal administrativo) tiene su propio registro de atención al usuario.

- En los turnos nocturnos solo se cuenta con una persona de Trabajo social para toda la institución, lo que les dificulta la continuidad para el registro y notificación de llegada del usuario.

Y para el indicador 2 (tiempo medio de permanencia en Admisión Continua), en el cual no se logró mantener el $100 \%$ de efectividad, puesto que se redujo a un $92.5 \%$, esta disminución de calidad se debió a que los servicios interconsultantes prolongaban la estancia del usuario en sala como vigilancia y observación para la estratificación de los pacientes.
Se realizaron reuniones con directivos médicos, Dirección operativa y administrativos, en las cuales se generaron acuerdos de propuestas de mejora de acuerdo a los resultados de la segunda medición:

- Registro en bitácora de solicitud de atención. Por Trabajo social y personal administrativo.

- Médico coordinador: personal médico y de enfermería en la notificación a personal de ventanilla destino del usuario; mantener la continuidad de las líneas de mejora establecidas.

- Reuniones con Servicios médicos interconsultantes: los pacientes no deberán permanecer más de ocho horas en el Servicio de Urgencias por causas atribuibles a la atención médica.

- Se acordó realizar una tercera medición para corroborar el seguimiento y adherencia a la implementación de las mejoras.

\section{ANÁLISIS DE LA TERCERA MEDICIÓN}

Se revisaron 48 casos, seleccionados aleatoriamente, encontrándose registro en todos los turnos, en el periodo comprendido entre el 22 de octubre y el 7 de noviembre del 2017.

Para el indicador 1, se obtuvo evidencia de calidad en el tiempo medio para la atención en Admisión Continua en $47 / 48$ casos, es decir, un $97.9 \%$ de efectividad frente al $100 \%$ esperado (Tabla 5). De los cuales $1 / 48$ $(2.08 \%)$ estuvo fuera del rango de tiempo de espera para atención establecido por triaje, con código 
Tabla 5. Tercera medición. Periodo: 22 de octubre al 7 de noviembre del 2017

\begin{tabular}{llcccc}
\hline $\begin{array}{l}\text { Parámetro Evidencia de calidad } \\
\text { (y excepciones o gestión critica) }\end{array}$ & \% esperado & $\begin{array}{c}\text { \% efectivo } \\
\text { medición 1 }\end{array}$ & $\begin{array}{c}\text { \% efectivo } \\
\text { medición } 2\end{array}$ & $\begin{array}{c}\text { \% efectivo } \\
\text { medición 3 }\end{array}$ \\
\hline 1 & Tiempo medio para la atención en Admisión Continua & $100 \%$ & 73.1 & 95.3 & 97.9 \\
2 & Tiempo medio de permanencia en Admisión Continua & $100 \%$ & 100 & 92.5 & 100 \\
\hline
\end{tabular}

Tabla 6. Codificación de triaje para la tercera medición

\begin{tabular}{lcc}
\hline Código & Casos en rango & Casos fuera de rango \\
\hline Inmediato & $1(2 \%)$ & \\
15 a 30 minutos & $12(25 \%)$ & $1(2 \%)$ \\
1 a 4 horas & $34(70.8 \%)$ & \\
\hline
\end{tabular}

amarillo (Tabla 6), demostrándose el aumento en la calidad de la atención con la capacitación continua.

Para el Indicador 2, se obtuvo evidencia de calidad en el tiempo medio de permanencia en Admisión Continua en 48/48 casos, es decir, una efectividad nuevamente del $100 \%$ (Tabla 5), resultado de la adherencia a las líneas de mejora de todo el personal que presta servicios a usuarios de Admisión Médica Continua.

\section{DISCUSIÓN}

En las múltiples sesiones de Comité de auditoría clínica, así como mesas de trabajo, con la finalidad de elegir tópicos adecuados para realizar auditorías clínicas, para los integrantes del SAMC fue de relevante importancia exponer la problemática que generaba la permanencia prolongada de pacientes en la sala de observación, por lo que en conjunto nos dedicamos a la búsqueda de herramientas necesarias para determinar los estándares que marquen los tiempos ideales compatibles con el objetivo institucional, así como una herramienta adecuada para su evaluación y de utilidad en la mejora con la reducción de los tiempos de espera para la atención y tiempos de permanencia en el servicio, señalando cuales son los factores modificables. Encontramos en nuestro marco jurídico los parámetros para la permanencia del paciente en el área de urgencias en la NOM-027-SSA3-20134, el periodo de estancia, y la Guía de Referencia Rápida Triaje Hospitalario de Primer Contacto en los Servicios de Urgencias de Adultos para el segundo y tercer nivel ${ }^{6}$ establece el tiempo de evaluación de codificación al contacto con el usuario. Sin embargo, en los indicadores de evaluación de la calidad de atención médica en nuestro sistema nacional de salud no se cuenta con indicadores de calidad que evalúen estos dos estándares de importancia relevante en la percepción de la calidad de atención para el usuario, por lo que adaptamos por medio de auditoría clínica la aplicación de los indicadores 1 (tiempo medio para la atención) y 2 (tiempo medio de permanencia) para Servicios de Urgencias al SAMC, que sí se realizan como tales en otros países, como España.

Logramos durante el proceso de las tres mediciones involucrar tanto a personal operativo como al administrativo, con el apoyo de los directivos, incluyendo en cada evaluación el compromiso de todo el personal operativo en cada turno. De esta manera logramos alcanzar un $100 \%$, la cifra esperada, en el indicador 2 (permanencia en Admisión Continua) y elevar considerablemente el indicador 1 (tiempo de espera para la atención), de un 73.1 a un $97.1 \%$ de efectividad, con una adecuada codificación de triaje, que se redujo de 11 de 41 casos fuera de rango para código amarillo a 1 de 48 casos.

\section{CONCLUSIONES}

Es de relevante importancia: mantener un registro continuo de usuarios para lograr realizar mediciones de autoevaluación, mantener la comunicación efectiva entre el personal involucrado en la atención de pacientes en el SAMC, y se debe establecer la cobertura de personal necesaria para la recepción y atención de usuarios. Se requiere compromiso mutuo entre directivos y personal operativo para seguimiento de reentrenamiento continuo.

La estandarización y medición de los indicadores de atención en las áreas críticas, como Admisión Médica 
Continua, por medio de auditoría clínica permiten conocer los procesos e identificar problemas, y de esta manera aplicar las acciones de mejora con efecto permanente.

En la implementación de las acciones para una mejor coordinación en los niveles de atención es necesaria la participación activa de todos los niveles de atención.

\section{BIBLIOGRAFÍA}

1. Catálogo de indicadores de atención médica de alta especialidad. IAMAE.

2. Grupo de Trabajo SEMES-Insalud. Calidad en los Servicios de Urgencias. Emergencias. 2001;13:60-5.
3. Conejo Gómez C, Falagán Mota JA, Ferrer Ivars R, López Domínguez 0 , Martínez de Pancorbo González C, Ramos Martín-Vegue AJ, et al.; Moral Iglesias L (coord.). Guía de gestión de los servicios de admisión continua y la documentación clínica. Instituto Nacional de la Salud. Subdirección General de Coordinación Administrativa. Madrid; 2000.

4. Alonso Ruiz-Luna M, Barrera Ávila JM, García Luque R, Garrido Cruz I, Ferrón García FJ, Jiménez Murillo L, et al.; Tejedor Fernández M (coord.). Manual de indicadores de actividad y calidad para urgencias y emergencias sanitarias. Junta de Andalucía. Consejería de Salud. Servicio Andaluz de Salud; 2000.

5. Guía de gestión de los servicios de admisión continua y la documentación clínica Guía de Referencia Rápida. Triaje hospitalario de primer contacto en los servicios de urgencias adultos para el segundo y tercer nivel. Guía de Práctica Clínica. Gobierno Federal de los Estados Unidos de México. Consejo de Salubridad General. ISSSTE-339-08.

6. NORMA Oficial Mexicana NOM-027-SSA3-2013, Regulación de los servicios de salud. Que establece los criterios de funcionamiento y atención en los servicios de urgencias de los establecimientos para la atención médica. DOF: 04/09/2013.

7. NORMA Oficial Mexicana NOM-206-SSA1-2002, Regulación de los servicios de salud. Que establece los criterios de funcionamiento y atención en vvlos servicios de urgencias de los establecimientos de atención médica. 